\title{
The enhanced recovery programme for management of patients following major colorectal resection at Furness General Hospital
}

\author{
Panna Patel, FRCS (Ed), FRCS (Gen Surg)
}

\section{INTRODUCTION}

Colorectal cancer is the second most common malignancy causing death in the western world. There are over 34,000 new cases and around 18,000 deaths each year in the UK. The only curative treatment is surgical resection. Traditionally, this has involved open surgery.

Enhanced recovery programme (ERP), so-called 'fast track' surgery, is a recent development in the surgical management of patients following major colorectal surgery. It was pioneered by Henrik Kehlet in Denmark. It is now implemented in the UK in many centres with great success. Recently, the government undertook to establish this programme in all hospitals in all specialities by 2012 .

ERP is a multimodal evidence-based structured approach to enhance patients' experience following surgery, shorten hospital stay and improve patient outcomes. The programme focuses on making sure the patients are active participants in their recovery. The aim of the programme is to reduce the stress response to surgical trauma with subsequent reduction in undesirable sequelae of surgical injury with improved recovery and reduction in postoperative morbidity and overall costs.(I)

The multimodal team approach involves a core team of surgeons, anaesthetists, colorectal nurse specialist, preoperative assessment nurses, dietician, physiotherapist, acute pain team, and ward nurses. The role of the anaesthetist is taken outside the operating theatre in the guise of an acute pain team, which provides supervision of complex analgesia regimens and advice to the ward nurses.

The main elements combine:

- patient education and optimisation pre-operatively

- modern anaesthetic, analgesic and surgical techniques to reduce the effects of surgical stress

- aggressive postoperative rehabilitation and ambulation

- early enteral feeding

This multimodal rehabilitation programme results in early return of the gut function, reduction in fatigue and shortened hospital stay when applied to open surgery.

\section{PRE-OPERATIVE COUNSELLING}

Patient participation is the key to success. Hence, patient education and motivation is very important. The patients are counselled with the various aspects of ERP and are encouraged to have a positive attitude and are reminded that most of them have a curable disease. The patients and their relatives are counselled about ERP by both the medical and nursing staff. They are provided with written information. A discharge date is agreed with the patients and their relatives. It is clear that the success of the programme lies in the patients taking charge of their recovery.

\section{PRE-OPERATIVE PREPARATION}

All patients undergo pre-operative assessment so that optimisation of any existing medical conditions may be carried out and any organ dysfunction identified. Bowel preparation using oral laxative is avoided in most cases so that patients are not dehydrated and do not have electrolyte disturbance prior to surgery. The patients are given high protein drinks for three days prior to surgery. In addition to this, high energy carbohydrate drinks (Preload) are given up to two hours prior to surgery. The result is that patients are non-fasted and well hydrated prior to surgery. All patients are admitted on day of surgery.

\section{SURGEON AND POSTOPERATIVE PERIOD}

The surgeons use transverse incisions where feasible. Nasogastric tube and drains are not used routinely. Catheters are removed early. Postoperatively, the patients are fed early, ie on the day of surgery a couple of hours after they are awake from the anaesthetic. Pain is controlled with epidural anaesthesia and intravenous paracetamol for 48 hours after surgery. The intravenous drips are removed on day one of surgery. Early mobilisation is encouraged from the day of surgery. Patients are encouraged to sit out for two hours on the day of surgery, and for eight hours from day one and walk 60 metres four times a day.

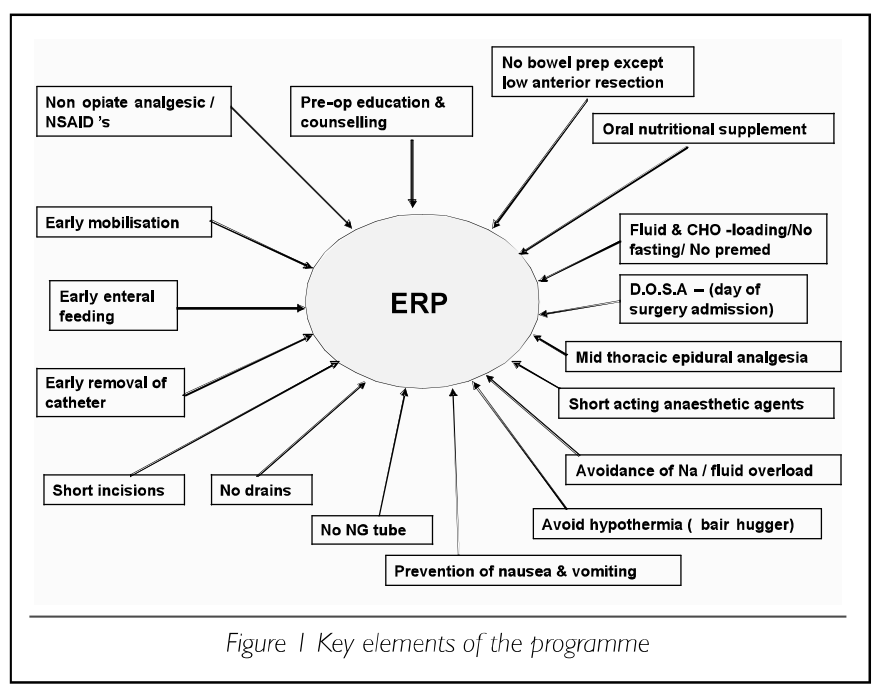




\section{THE PERI-OPERATIVE PERIOD}

The anaesthetist's view (Dr Jyrki Karvonen, consultant anaesthetist, Furness General Hospital)

The aim of anaesthesia is to support ERP by providing good pain relief rapid emergence from anaesthesia avoiding postoperative nausea and vomiting (PONV), and allowing the patient's early mobilisation.

Fluid management during operation is individualised using esophageal Doppler monitoring (CardioQ). It has been shown to reduce length of stay in hospital, (23) which again reduces the total cost of the operation. This is crucial in today's bankrupt world. With esophageal Doppler cardiac, output and stroke volume are optimised and fluids can be given according the patient's needs, ${ }^{\left({ }^{4}\right)}$ hopefully preventing postoperative tissue oedema in the operation area and guaranteeing rapid bowel function. Delivery of oxygen to the gut is thus guaranteed and with superfluous inspiratory fraction of oxygen (FiO2) 60-65\% anaerobic bacteria contamination is combated. Mean arterial pressure is kept over $70 \mathrm{mmHg}$ to ensure proper tissue perfusion

In this series we have used lower thoracic epidural anaesthesia (TEA), which is inserted and activated when the patient is awake to guarantee its proper function. Initial epidural bolus of up to $20 \mathrm{ml}$ bupivacaine and lo0ug of fentanyl is given in 2-3 increments before induction. Epidural infusion with $0.1 \%$ bupivacaine and $2 \mathrm{ug} / \mathrm{ml}$ fentanyl is started within the next hour (4-8ml/hour). In most patients, pain relief peri-operatively is very good and the patient can even be mobilised later in the same day. Postoperative opioids, eg morphine which is a major component in causing PONV, can be avoided with TEA

The patient is kept warm and central temperature is measured and controlled with a hot air circulating blanket to avoid the hypothermia that may complicate open abdominal surgery.

Rapid emergence from anaesthesia in this series was obtained with infusions of the anaesthetic agent propofol and the ultrashort-acting opioid remifentanil.

Postoperative nausea and vomiting are potential complications that will delay recovery: they are reduced in this series by the use of propofol and the avoidance of volatile anaesthesics and nitrous oxide during surgery..$^{(5.6)}$ Muscle relaxation is allowed to wear off and the anticholinesterase neostigmine, a cause of PONV, is not used to reverse it. Pharmacological prevention of PONV is achieved with a mixture of dexamethasone, ondansetron and cyclizine.

Pain relief with TEA reduces postoperative ileus and the stress response to surgery by providing a block (levels T6-TI2) to the sympathetic innervation of the celiac and mesenteric axes and the adrenal medulla, () and is continued to the second postoperative day, supervised by the acute pain team. Regular paracetamol is given to support postoperative pain relief.

Anaesthesia thus supports the body's homeostasis and fulfils the requirements for the patient's rapid discharge in ERP.

\section{DISCHARGE}

We aim to discharge patients who have had colorectal resections on day four after their surgery. Patients with a stoma stay longer (aim for seven days) as they have to be trained in the use of a stoma. The discharge criteria remain the same as before, ie gut function is returned (no nausea, tolerating oral diet and bowel functioning normally), pain is controlled, patients are independently mobile, and patients are happy to be discharged. All patients on discharge are given the contact name of the colorectal nurse specialist, contact numbers, and open access to the ward for one week in event of any problems. A review appointment to discuss the histology is made in a week's time.

\section{ESTABLISHMENT OF ERP AT FURNESS GENERAL HOSPITAL (FGH)}

This programme has successfully been established at FGH since October 2007. A retrospective audit of the patients undergoing major colorectal surgery within an enhanced programme at FGH was carried out. The multimodal rehabilitation programme has improved patient recovery and reduced hospital stay.

\section{Methodology}

Data was collected on 38 consecutive patients undergoing major colorectal resection from October 2007 to September 2008 using a proforma designed for retrospective data collection and the consultant surgeon's database. Six patients were excluded due to complex surgery, eg gastrectomy, abdominoperineal resections. Analysis of data was carried out on 32 patients. Length of stay was the primary endpoint.

\section{Results}

Clinical outcomes in 32 patients were analysed. The age range was between 56 to 90 , with a median of 73 years. There were 14 females and 18 males. The mean body mass index (BMI) was 27 (20-36).

\section{Hospital stay}

The length of stay was four days in over a third $(37 \%)$ of patients; and by day six, two thirds (60\%) were discharged home. The median length of stay was six days.

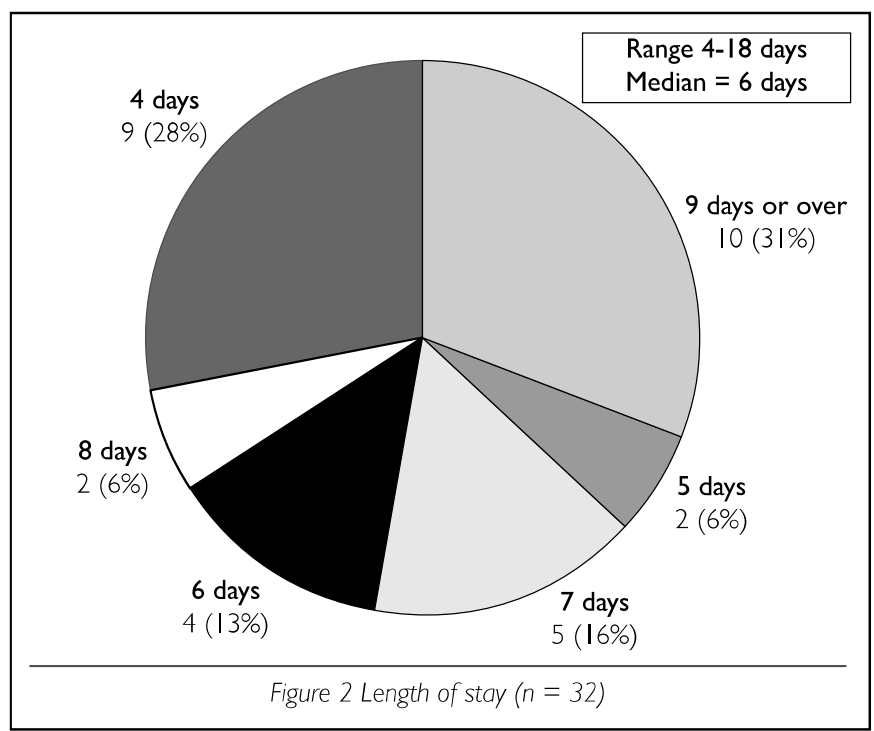

Gastrointestinal function

There was early return of gut function with $90 \%$ of patients having solid diet on day one, $60 \%$ passed flatus on day one, and $90 \%$ by day three (see figure 3 ). By day two, nearly half (47\%) of the patients had opened their bowels, increasing to $94 \%$ by day four (see figure 4).

\section{Morbidity and mortality}

There were no major complications and none of the patients required a re-operation. There were minor complications in six patients, such as retention of urine, ileus and wound infection. Only two patients were readmitted with minor complaints. There were no deaths in this series.

\section{DISCUSSION}

The introduction of ERP has transformed postoperative care following major colorectal surgery. The national average of stay following major colorectal surgery is 14 days. With ERP there is significant reduction in length of hospital stay to two to five 


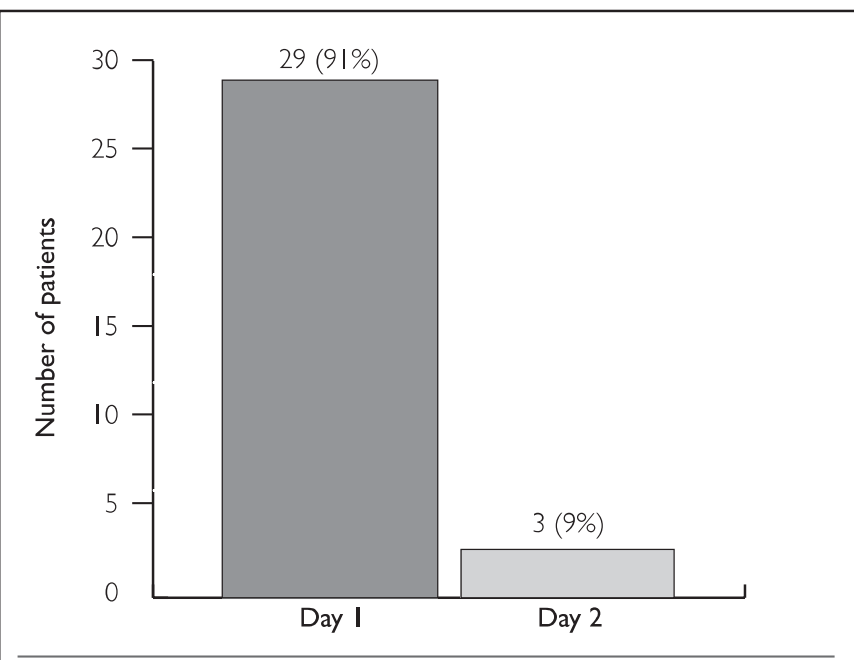

Figure 3 Oral diet - gut function post-op, solid food $(n=32)$

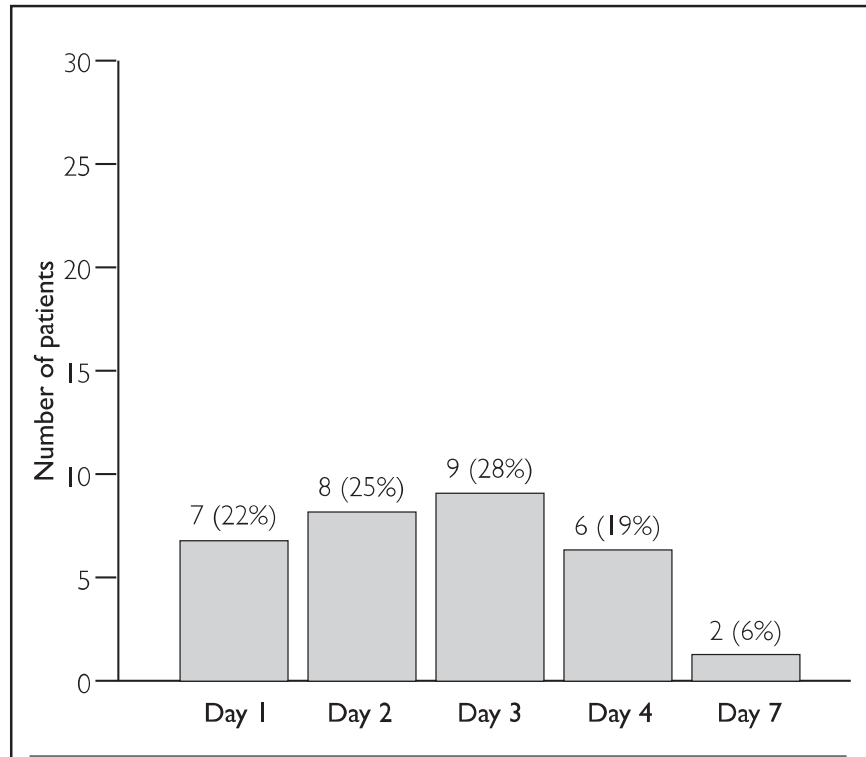

Figure 4 Bowel function - gut function post-op, bowels opened $(n=32)$

days following major colorectal surgery. ${ }^{(8,9)}$ In addition, there are many benefits in the form of early return of gut function with reduced ileus, reduced morbidity. The early normalisation of gastrointestinal function allows early oral nutrition which otherwise has been demonstrated to improve postoperative outcome. ${ }^{(10)}$

This programme encourages a multidisciplinary and team approach to patient care among medical and nursing staff. There is a clear improvement in the postoperative care delivered to patients. The main concept of 'fast track' surgery is to reduce the postoperative stress following major surgery by a multimodal approach with the hope of improving surgical outcome.

The team has promoted this approach to other specialties through a series of lectures using the results from the audit to generate interest and promote uptake. We are actively involved in establishing ERP in colorectal surgery at Royal Lancaster Infirmary.

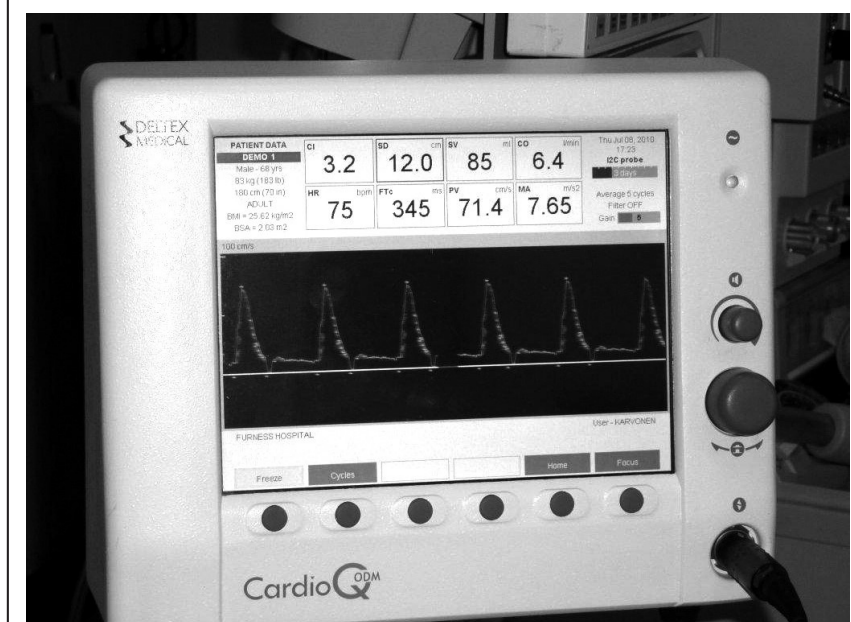

Non-invasive cardiac output measurements enable the anaesthetist to titrate fluid infusions to maximum effect, sparing the dangers of fluid overload and the consequent tissue oedema. It has a role to play in the enhanced recovery programme for bowel surgery.

\section{CONCLUSION}

Our results have demonstrated that ERP provided to patients having open major colorectal surgery is feasible and safe to improve patient care. It improves the patient journey, results in early return of gut function, early mobilisation and reduced the length of stay in hospital without adverse effects. The functional recovery is rapid after major colorectal resection with a multimodal approach. We have established ERP at FGH with the available resources and without additional cost to the trust.

\section{FUTURE DEVELOPMENT}

The colorectal team is now involved in establishing a laparoscopic colorectal resection service and with this surgical trauma will be further reduced. Application of the principles of ERP to these patients will add to their speedy recovery and with this further reduce hospital stay.

\section{ACKNOWLEDGEMENTS}

I would like to thank the members of our 'team'. Of the nursing staff, in particular: Carol Park, modern matron, and Suzanne Thompson, colorectal nurse specialist. My anaesthesia colleagues: Dr Jyrki Karvonen and Dr Gill O'Connell, who established the anaesthetic and postoperative pain management protocols. The departments of physiotherapy and dietetics cheerfully cooperated with my plans to build a team without additional resources. I would also like to express my gratitude to the Audit department who have supported and helped to promote ERP at FGH and cross bay.

\section{REFERENCES}

I. Kehlet H. Multimodal approach to control postoperative pathophysiology and rehabilitation. $\mathrm{Br}$ J Anaesth 1997;78(5):606-7 
2. Phan TD, Ismail H, Heriot AG, Ho KM. Improving perioperative outcomes: fluid optimization with the esophageal Doppler monitor, a metaanalysis and review. J Am Coll Surg 2008;207(6):935-4 |

3. Hamilton M, Grocott PW, Mythen M, Bennett. Does oesophageal Doppler guided goal directed therapy reduce surgical mortality and length of stay? Intensive Care Med 2006;32(SI3):SII 17

4. Wakeling HG, McFall MR, Jenkins CS, et al. Intra-operative oesophageal Doppler guided fluid management shortens postoperative hospital stay after major bowel surgery. $\mathrm{Br}$ J Anaesth 2005;95:634-42

5. Zingg U, Miskovic D, Hamel CT, Erni L, Oertli D, Metzger U. Influence of thoracic epidural analgesia on postoperative pain relief and ileus after laparoscopic colorectal resection: benefit with epidural analgesia. Surg Endosc 2009;23(2):276-82. Epub 2008 Mar 25

6. Jottard KJC, van Berlo C, Jeuken L, Dejong C; on behalf of the ERAS group. Changes in outcome during implementation of a fast-track colonic surgery project in a university-affiliated general teaching hospital: advantages reached with ERAS over a I-year period. Dig Surg 2008;25(5):335-8
7. Vari A, Gazzanelli S, Cavallaro G, et al. Post-operative nausea and vomiting (PONV) after thyroid surgery: a prospective, randomized study comparing totally intravenous versus inhalational anesthetics. Am Surg 2010;76(3):325-8

8. Basse $L$, Jackobsen $D H$, Kehlet $H$. A clinical pathway to accelerate recovery after colonic resection. Ann Surg 2000;232( 1$): 51-7$

9. King PM, Blazeby JM, Ewings P, et al. Randomized clinical trial comparing laparoscopic and open surgery for colorectal cancer within an enhanced recovery programme. Br J Surg 2006;93(3);300-8

10. Lewis SJ, Egger M, Sylvester PA, Thomas S. Early enteral feeding versus "nil by mouth" after gastrointestinal surgery: systematic review and meta-analysis of controlled trials. Br Med J 200।;323(73।6):773-7

\section{MBMJ Prize for best article by a junior doctor (2009)}

\section{Congratulations to Roanna Mitford. Her article}

'Audit into the effectiveness of self-help groups', published in

Spring 2009, Volume 5 Number 10, wins the prize,

which is worth $£ 200$ and is awarded annually. 